\title{
MULTIPLE SOLUTIONS FOR THE $p$-LAPLACIAN UNDER GLOBAL NONRESONANCE
}

\author{
MANUEL A. DEL PINO AND RAUUL F. MANÁSEVICH
}

(Communicated by Kenneth R. Meyer)

\begin{abstract}
Via the study of a simple Dirichlet boundary value problem associated with the one-dimensional $p$-Laplacian, $p>1$, we show that in globally nonresonant problems for this differential operator the number of solutions may be arbitrarily large when $p \in(1, \infty) \backslash\{2\}$. From this point of view $p=2$ turns out to be a very special case.
\end{abstract}

\section{INTRODUCTION}

Let us consider the boundary value problem

$$
\begin{gathered}
\left(\left|u^{\prime}\right|^{p-2} u^{\prime}\right)^{\prime}+f\left(|u|^{p-2} u\right)=h(x), \quad x \in(0, T), \\
u(0)=u(T)=0,
\end{gathered}
$$

where $f \in C(\mathbf{R}, \mathbf{R})$ and $h \in C[0, T]$. In (1.1) and henceforth ${ }^{\prime}=d / d x$ and $1<p<\infty$. It follows from the results of [2], (see also [3]), that (1.1)(1.2) possesses at least one solution if $f$ satisfies the asymptotic nonresonance condition

$$
\lambda_{k}<\liminf _{|s| \rightarrow \infty} f(s) / s \leq \limsup _{|s| \rightarrow \infty} f(s) / s<\lambda_{k+1}
$$

for some $k \in \mathbf{N}$. In (1.3), $\left\{\lambda_{n}\right\}_{n=1}^{\infty}$ is the sequence of eigenvalues corresponding to the problem

$$
\begin{gathered}
\left(\left|u^{\prime}\right|^{p-2} u^{\prime}\right)^{\prime}+\lambda|u|^{p-2} u=0, \quad x \in(0, T), \\
u(0)=u(T)=0 .
\end{gathered}
$$

These eigenvalues are given by

$$
\lambda_{k}=\left(\frac{k \pi_{p}}{T}\right)^{p}, \quad k \in \mathbf{N},
$$

Received by the editors November 1, 1989 and, in revised form, January 16, 1990.

1980 Mathematics Subject Classification (1985 Revision). Primary 34B15; Secondary 34B10.

Key words and phrases. Nonresonance, multiple solutions.

This research was sponsored by the FONDECYT, Research grant 0546-88 and by the DTI, Univ. de Chile. 
where

$$
\pi_{p}=2(p-1)^{1 / p} \int_{0}^{1} \frac{d s}{\left(1-s^{p}\right)^{1 / p}}
$$

(see [5], [6] or [3]).

We note that condition (1.3) is satisfied if, e.g., $f$ is of class $C^{1}$ and there exist constants $\alpha, \beta$ such that

$$
\lambda_{k}<\alpha \leq f^{\prime}(s) \leq \beta<\lambda_{k+1}
$$

for some $k \in \mathbf{N}$ and all $s \in \mathbf{R}$.

It is well known that in the case $p=2$, the global nonresonance condition (1.8) also ensures uniqueness of the solution of (1.1), (1.2). At this point a natural question arises. Given $p \in(1, \infty)$, does $(1.8)$ suffice for the unique solvability of (1.1), (1.2)? Our aim in this paper is to show that the answer is negative if $p \neq 2$. To do this, the rest of this paper will consider the boundary value problem

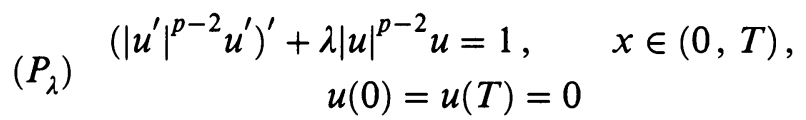

which is obtained from (1.1), (1.2) by setting $f(s)=\lambda s, s \in \mathbf{R}$, and $h \equiv 1$.

We note that in problem $\left(P_{\lambda}\right)$ the nonresonance condition (1.8) holds if and only if $\lambda_{k}<\lambda<\lambda_{k+1}$ for some $k \in \mathbf{N}$.

Next let us denote the number of solutions of $\left(P_{\lambda}\right)$ by $N_{p}(\lambda)$. For $p=2$, that is, for the linear case, elementary calculations show that

$$
N_{2}(\lambda)= \begin{cases}1 & \text { if } \lambda \neq(k \pi / T)^{2} \text { for all } k \in \mathbf{N}, \\ 0 & \text { if } \lambda=((2 k-1) \pi / T)^{2} \text { for some } k \in \mathbf{N}, \\ \infty & \text { if } \lambda=(2 k \pi / T)^{2} \text { for some } k \in \mathbf{N}\end{cases}
$$

Thus, for $p=2$ and $\lambda$ between consecutive eigenvalues, $N_{2}(\lambda)=1$.

We will show in the next section that for $p \neq 2$, the number of solutions of $\left(P_{\lambda}\right)$ can be arbitrarily large. Indeed, and as a consequence of our Theorem 2.1 we will obtain

$$
\lim _{\lambda \rightarrow \infty} N_{p}(\lambda)=\infty
$$

In particular, for $p \neq 2$ and $\lambda$ between consecutive eigenvalues, not only may $N_{p}(\lambda)$ be different from one but also $\lambda$ large implies $N_{p}(\lambda)$ large. Thus, concerning $N_{p}(\lambda)$ there is a sharp contrast between problem $\left(P_{\lambda}\right)$ under the nonresonant condition (1.8) for $p \neq 2$ and the case $p=2$.

These findings reveal that the uniqueness problem for (1.1)-(1.2) under global nonresonance can be highly nontrivial.

Remark. If we let $f(0)=0, h \equiv 0$ in (1.1), (1.2) then (1.8) ensures that $u \equiv 0$ is the unique solution of (1.1), (1.2). This result is easily shown from Sturm's theorem for equations of the form

$$
\left(\left|u^{\prime}\right|^{p-2} u^{\prime}\right)^{\prime}+a(x)|u|^{p-2} u=0
$$


(see for example [4]), and the fact that an eigenfunction associated with $\lambda=\lambda_{k}$ in (1.4), (1.5) possesses exactly $k-1$ zeros in $(0, T)$.

\section{MAIN RESUlt AND CONSEQUENCES}

In this section we state our main result, Theorem 2.1 , and derive some consequences from it. Theorem 2.1 will be proved in the next section.

Together with the sequence $\left\{\lambda_{k}\right\}_{k=1}^{\infty}$ of eigenvalues of $(1.4),(1.5)$ we will consider the sequence $\left\{\mu_{k}\right\}_{k=1}^{\infty}$ defined by

$$
\mu_{k}=\left(k p^{\prime} \pi_{p} / T\right)^{p}, \quad k \in \mathbf{N} \text {. }
$$

In (2.1) and henceforth, $p^{\prime}=p /(p-1)$. We observe that $\mu_{k}<(=)(>) \lambda_{2 k}$ if $p<(=)(>) 2$. These numbers $\mu_{k}, k \in \mathbf{N}$, will play an important role in our results. Indeed, it is their nonuniform distribution with respect to the $\lambda_{k}$ 's for $p \neq 2$ which produces the existence of a large number of solutions to $\left(P_{\lambda}\right)$ for large $\lambda$.

We will say that a function $u \in C^{1}[0, T]$ belongs to $E_{k}^{+}\left(E_{k}^{0}\right)\left(E_{k}^{-}\right)$if $u$ possesses exactly $k-1$ zeros in $(0, T)$ and $u^{\prime}(0)>(=)(<) 0$.

Theorem 2.1. (a) If $\lambda \in\left(0, \lambda_{1}\right)$, then $\left(P_{\lambda}\right)$ possesses exactly one solution $u$, and $u \in E_{1}^{-}$.

(b) If $\lambda=\lambda_{1}$, then $\left(P_{\lambda}\right)$ has no solution.

(c) If $\lambda$ is strictly between $\lambda_{2 k-1}$ and $\mu_{k}$, then $\left(P_{\lambda}\right)$ possesses at least one solution $u \in E_{2 k-1}^{+}$.

(d) If $\lambda$ is strictly between $\mu_{k}$ and $\lambda_{2 k+1}$, then $\left(P_{\lambda}\right)$ possesses at least one solution $u \in E_{2 k+1}^{+}$.

(e) If $\lambda=\mu_{k}$ then $\left(P_{\lambda}\right)$ possesses a solution $u \in E_{k}^{0}$.

(f) If $\lambda$ is strictly between $\mu_{k}$ and $\lambda_{2 k}$, then $\left(P_{\lambda}\right)$ possesses a solution $u$ in $E_{2 k}^{+}$and a solution $v$ in $E_{2 k}^{-}$.

Remark. In contrast with (a) of Theorem 2.1, it is shown in [3] that for $p>2$ and $\lambda \in\left(0, \lambda_{1}\right)$ one can always find an $h \in C[0, I]$ such that the problem

$$
\begin{gathered}
\left(\left|u^{\prime}\right|^{p-2} u^{\prime}\right)^{\prime}+\lambda|u|^{p-2} u=h(x), \quad x \in(0, T), \\
u(0)=u(T)=0
\end{gathered}
$$

admits at least two solutions. We also remark that Anane and Gossez [1] have studied the resonance-nonresonance problem for the $p$-Laplacian in the case where the nonlinearity "lies" to the left of the first eigenvalue.

By combining (a)-(f) of Theorem 2.1 we can easily obtain the following.

Corollary 2.2. Let $p \in(1, \infty) \backslash\{2\}$. Then $\left(P_{\lambda}\right)$ is solvable for all $\lambda>0$ except $\lambda=\lambda_{1}$ and, eventually, those numbers $\lambda$ of the form $\lambda=\lambda_{2 k-1}$ for $k<$ $1 /\left|p^{\prime}-2\right|$.

From this corollary we obtain, in particular, that $\left(P_{\lambda}\right)$ is solvable for all large positive $\lambda$. Furthermore, as $\lambda$ goes to infinity the number of solutions $N_{p}(\lambda)$ of $\left(P_{\lambda}\right)$ goes to infinity, as the following estimate shows. 
Proposition 2.3. Let $p \in(1, \infty), p \neq 2$. Then the number of solutions $N_{p}(\lambda)$ of $\left(P_{\lambda}\right)$ satisfies

$$
N_{p}(\lambda) \geq \frac{3 T \lambda^{1 / p}}{\pi_{p}}\left|\frac{1}{p^{\prime}}-\frac{1}{2}\right|-3
$$

for all $\lambda>0$. In particular, $\lim _{\lambda \rightarrow \infty} N_{p}(\lambda)=\infty$.

Proof. We will assume $p>2$. The case $p<2$ can be treated similarly.

Let us fix $\lambda>0$ and denote by $M_{j}$ the number of positive integers such that (f) of Theorem 2.1 holds, i.e.,

$$
M_{f}=\operatorname{card}\left\{k \in \mathbf{N} \mid \frac{p^{\prime} \pi_{p} k}{T}<\lambda^{1 / p}<\frac{2 \pi_{p} k}{T}\right\} .
$$

Clearly

$$
M_{f} \geq \max \left\{k \in \mathbf{N} \mid k<\frac{T \lambda^{1 / p}}{p^{\prime} \pi_{p}}\right\}-\min \left\{k \in \mathbf{N} \mid \frac{T \lambda^{1 / p}}{2 \pi_{p}}<k\right\}+1
$$

and hence

$$
M_{f} \geq\left(\frac{T \lambda^{1 / p}}{p^{\prime} \pi_{p}}-1\right)-\left(\frac{T \lambda^{1 / p}}{2 \pi_{p}}+1\right)=\frac{T \lambda^{1 / p}}{\pi_{p}}\left(\frac{1}{p^{\prime}}-\frac{1}{2}\right)-1 .
$$

Next, let us denote by $M_{c}\left(M_{d}\right)$ the number of positive integers such that (c) ((d)) of Theorem 2.1 holds. Estimates similar to those for $M_{f}$ yield

$$
\begin{aligned}
& M_{c} \geq \frac{T \lambda^{1 / p}}{\pi_{p}}\left(\frac{1}{p^{\prime}}-\frac{1}{2}\right)-\frac{3}{2}, \\
& M_{d} \geq \frac{T \lambda^{1 / p}}{\pi_{p}}\left(\frac{1}{p^{\prime}}-\frac{1}{2}\right)-\frac{1}{2} .
\end{aligned}
$$

From (2.5)-(2.7) we obtain

$$
N_{p}(\lambda) \geq M_{c}+M_{d}+m_{f} \geq \frac{3 T \lambda^{1 / p}}{\pi_{p}}\left(\frac{1}{p^{\prime}}-\frac{1}{2}\right)-3,
$$

and hence the proposition.

\section{Proof of Theorem 2.1}

In this section we will prove Theorem 2.1. To this end, we will first study some properties of the solution to the initial value problem

$$
\begin{gathered}
\left(\left|u^{\prime}\right|^{p-2} u^{\prime}\right)^{\prime}+\lambda|u|^{p-2} u=1, \\
u(0)=0 \quad u^{\prime}(0)=\alpha .
\end{gathered}
$$


We will construct a global solution to this problem. Multiplying both sides of (3.1) by $u^{\prime}$ and integrating from 0 to $t$ we find that a solution $u$ to (3.1), (3.2) must satisfy the energy relation

$$
\frac{\left|u^{\prime}(t)\right|^{p}}{p^{\prime}}+\lambda \frac{|u(t)|^{p}}{p}=\frac{|\alpha|^{p}}{p^{\prime}}+u(t) .
$$

Let $t_{\lambda}(\alpha)$ be the first positive zero of $u^{\prime}$. Thus for $t \in\left(0, t_{\lambda}(\alpha)\right)$

$$
t=\int_{0}^{u(t)} d w /\left(\alpha^{p}+p^{\prime} w-\lambda \frac{w^{p}}{p-1}\right)^{1 / p}
$$

if $\alpha \geq 0$, and

$$
t=\int_{0}^{-u(t)} d w /\left(|\alpha|^{p}-p^{\prime} w-\lambda \frac{w^{p}}{p-1}\right)^{1 / p}
$$

if $\alpha<0$. Thus, considering the function

$$
F(s)=\int_{0}^{s} d w /\left(|\alpha|^{p}+p^{\prime} \operatorname{sgn}(\alpha) w-\lambda \frac{w^{p}}{p-1}\right)^{1 / p},
$$

where

it follows that

$$
\operatorname{sgn}(\alpha)= \begin{cases}1 & \text { if } \alpha \geq 0 \\ -1 & \text { if } \alpha<0\end{cases}
$$

$$
t_{\lambda}(\alpha)=F(q(\alpha)),
$$

where $q(\alpha)$ is the unique positive root of the equation

$$
\lambda x^{p} /(p-1)-p^{\prime} \operatorname{sgn}(\alpha) x=|\alpha|^{p} .
$$

Also, from (3.4)-(3.6) and for $t \in\left(0, t_{\lambda}(\alpha)\right]$, we have

$$
u(t)= \begin{cases}F^{-1}(t) & \text { if } \alpha \geq 0, \\ -F^{-1}(t) & \text { if } \alpha<0 .\end{cases}
$$

Conversely, if we have a function $u$ of the form (3.9) it can be directly verified that $u$ satisfies (3.1), (3.2) and hence is the unique solution of this initial value problem on the interval $\left(0, t_{\lambda}(\alpha)\right]$ with $t_{\lambda}(\alpha)$ defined by (3.7).

Next, let us extend $u$ to obtain a global solution $u_{\lambda}(\alpha, t)$ to (3.1), (3.2). Thus define $u_{\lambda}(\alpha, t)=u(t)$ for $t \in\left(0, t_{\lambda}(\alpha)\right], u_{\lambda}(\alpha, t)=u\left(2 t_{\lambda}(\alpha)-t\right)$ for $t \in\left[t_{\lambda}(\alpha), 2 t_{\lambda}(\alpha)\right]$, and $u_{\lambda}(\alpha, t)=u_{\lambda}\left(-\alpha, 2\left(t_{\lambda}(\alpha)+t_{\lambda}(-\alpha)\right)-t\right)$ for $t \in\left[2 t_{\lambda}(\alpha)\right.$, $\left.2\left(t_{\lambda}(\alpha)+t_{\lambda}(-\alpha)\right)\right]$. Finally we periodically extend this function to the whole real line in a $2\left(t_{\lambda}(\alpha)+t_{\lambda}(-\alpha)\right)$-periodic manner. It is easily verified that $u_{\lambda}(\alpha, t)$ is of class $C^{1}$ and solves (3.1), (3.2). It can be shown that this is actually the unique solution of (3.1), (3.2).

We note that the zeros of $u_{\lambda}(\alpha, t)$ are the numbers $2 k t_{\lambda}(\alpha)+2(k-\varepsilon) t_{\lambda}(-\alpha)$, $k \in \mathbf{N}, \varepsilon=0$ or 1 . Clearly, whenever one of these numbers equals $T$ we obtain a solution of $\left(P_{\lambda}\right)$.

Next, let us show some properties of the function $t_{\lambda}(\alpha)$ which will be needed in the proof of Theorem 2.1. 
Lemma 3.1. The function $t_{\lambda}(\alpha)$ satisfies

(a) $t_{\lambda}$ is strictly decreasing and continuous on the intervals $(-\infty, 0)$ and $[0, \infty)$.

(b) $t_{\lambda}$ presents a jump discontinuity at $\alpha=0$; more precisely,

$$
0=\lim _{\alpha \rightarrow 0^{-}} t_{\lambda}(\alpha)<t_{\lambda}(0)=p^{\prime} \pi_{p} / 2 \lambda^{1 / p} .
$$

(c)

$$
\lim _{\alpha \rightarrow-\infty} t_{\lambda}(\alpha)=\pi_{p} / 2 \lambda^{1 / p}=\lim _{\alpha \rightarrow+\infty} t_{\lambda}(\alpha) .
$$

Proof. From (3.6) and (3.7) follows

$$
t_{\lambda}(\alpha)=\int_{0}^{q(\alpha)} d w /\left(|\alpha|^{p}+p^{\prime} \operatorname{sgn}(\alpha) w-\lambda \frac{w^{p}}{p-1}\right)^{1 / p} .
$$

Substituting $w=s q(\alpha)$ in (3.12) and calling on (3.8) with $x=q(\alpha)$ we obtain

$$
t_{\lambda}(\alpha)=\int_{0}^{1} d s /\left(-\frac{1}{q(\alpha)^{p-1}} p^{\prime} \operatorname{sgn}(\alpha)(1-s)+\frac{\lambda}{p-1}\left(1-s^{p}\right)\right)^{1 / p} .
$$

To examine the behavior of $q(\alpha)$ with respect to $\alpha$ we note that the Implicit Function Theorem and (3.8) imply that $q(\alpha)$ is of class $C^{1}$ on $\mathbf{R} \backslash\{0\}$ and

$$
\frac{d q}{d \alpha}(\alpha)=\frac{p|\alpha|^{p-2} \alpha}{p^{\prime}\left(\lambda q(\alpha)^{p-1}-\operatorname{sgn}(\alpha)\right)} .
$$

From the definition of $q(\alpha)$, we easily see that the denominator on the righthand side of (3.14) is positive and hence that $q(\alpha)$ is strictly increasing and continuous for $\alpha \in[0, \infty)$ and strictly decreasing and continuous for $\alpha \in$ $(-\infty, 0)$. Thus (a) follows immediately from (3.13).

To show (b) we first observe that $q\left(0^{-}\right)=0$ and hence from (3.12) we conclude that $\lim _{\alpha \rightarrow 0^{-}} t_{\lambda}(\alpha)=0$. Next, setting $\alpha=0$ in (3.13) and using the fact that $q(0)=(p / \lambda)^{1 /(p-1)}$ from (3.8), we obtain

$$
t_{\lambda}(0)=\left(\frac{p-1}{\lambda}\right)^{1 / p} \int_{0}^{1} \frac{d s}{\left(s-s^{p}\right)^{1 / p}} .
$$

The substitution $s=\tau^{p^{\prime}}$ in (3.15) yields

$$
t_{\lambda}(0)=\left(\frac{p-1}{\lambda}\right)^{1 / p} \int_{0}^{1} \frac{p^{\prime} d \tau}{\left(1-\tau^{p}\right)^{1 / p}}=\frac{p^{\prime} \pi_{p}}{2 \lambda^{1 / p}} .
$$

This shows (b). Finally, to show (c), we note that $q(\alpha) \rightarrow+\infty$ as $|\alpha| \rightarrow \infty$. Letting $\alpha$ go to $\pm \infty$ in (3.15), it follows from the Dominated Convergence Theorem that

$$
\lim _{\alpha \rightarrow \pm \infty} t_{\lambda}(\alpha)=\left(\frac{p-1}{\lambda}\right)^{1 / p} \int_{0}^{1} \frac{d s}{\left(1-s^{p}\right)^{1 / p}}=\frac{\pi_{p}}{2 \lambda^{1 / p}} .
$$

This concludes the proof of $(c)$ and hence the lemma. 
With these preliminaries we are now ready to prove Theorem 2.1. For notational simplicity, throughout the proof we will assume $T=\pi_{p}$. In this case $\lambda_{k}=k^{p}$ and $\mu_{k}=\left(p^{\prime} k\right)^{p}, k \in \mathbf{N}$.

Proof of Theorem 2.1. (a) Assume that $0<\lambda<1$. Then from Lemma 2.1 we find $2 t_{\lambda}(\alpha)>\pi_{p}$ for $\alpha \geq 0$. Hence there is no solution of $\left(P_{\lambda}\right)$ with nonnegative derivative at $t=0$. Again from Lemma 3.1, but for $\alpha<0$, we see that there is a unique $\alpha^{*}<0$ such that $2 t_{\lambda}\left(\alpha^{*}\right)=\pi_{p}$. It follows that $u_{\lambda}\left(\alpha^{*}, t\right)$ is the unique solution of $\left(P_{\lambda}\right)$ and it belongs to $E_{1}^{-}$.

(b) Suppose that $\lambda=1$. The absence of solutions to $\left(P_{\lambda}\right)$ in this case follows directly from the facts that $2 t_{\lambda}(\alpha)>\pi_{p}$ for $\alpha \geq 0$, and $2 t_{\lambda}(\alpha)<\pi_{p}$ for $\alpha<0$.

(c) We assume $\lambda$ strictly between $\lambda_{2 k-1}$ and $\mu_{k}$, i.e., $\lambda^{1 / p}$ is between $2 k-1$ and $p^{\prime} k$. Let us consider the function

$$
f(\alpha)=2(k-1)\left(t_{\lambda}(\alpha)+t_{\lambda}(-\alpha)\right)+2 t_{\lambda}(\alpha) .
$$

From Lemma 3.1 we see that $f$ is continuous on $(0, \infty), f\left(0^{+}\right)=p^{\prime} k \pi_{p} / \lambda^{1 / p}$, and $\lim _{\alpha \rightarrow+\infty} f(\alpha)=(2 k-1) \pi_{p} / \lambda^{1 / p}$. Now, the fact that $\lambda^{1 / p}$ is strictly between $2 k-1$ and $p^{\prime} k$ implies the existence of $\bar{\alpha}>0$ such that $f(\bar{\alpha})=\pi_{p}$, and hence $u(t) \equiv u_{\lambda}(\bar{\alpha}, t)$ is a solution to $\left(P_{\lambda}\right)$ with exactly $2 k-2$ inner zeros. Clearly $u \in E_{2 k-1}^{+}$.

(d) The proof is analogous to that of (c) except that this time we consider the function

$$
f(\alpha)=2 k\left(t_{\lambda}(\alpha)+t_{\lambda}(-\alpha)\right)+2 t_{\lambda}(\alpha)
$$

on the interval $(-\infty, 0)$.

(e) If $\lambda=\mu_{k}$, then $\lambda^{1 / p}=k p^{\prime}$. This implies that $2 k t_{\lambda}(0)=\pi_{p}$ and, therefore, $u(t) \equiv u_{\lambda}(0, t)$ is a solution of $\left(P_{\lambda}\right)$ with exactly $k-1$ inner zeros. Clearly $u \in E_{k}^{0}$. We note that in this case all the zeros of $u$ are double.

(f) Suppose finally that $\lambda$ is strictly in between $k p^{\prime}$ and $2 k$. For this case we define

$$
f(\alpha)=2 k\left(t_{\lambda}(\alpha)+t_{\lambda}(-\alpha)\right) .
$$

Clearly $f$ is continuous on each of the intervals $(-\infty, 0)$ and $(0, \infty)$. Also $f\left(0^{+}\right)=p^{\prime} k \pi_{p} / \lambda^{1 / p}$ and $\lim _{\alpha \rightarrow \pm \infty} f(\alpha)=2 k \pi_{p} / \lambda^{1 / p}$. Reasoning as before we obtain the existence of solutions $u, v$ of $\left(P_{\lambda}\right)$ with $u \in E_{2 k}^{+}$and $v \in E_{2 k}^{-}$. This concludes the proof of the theorem.

\section{REFERENCES}

1. A. Anane and J. P. Gossez, Strongly nonlinear elliptic problems near resonance: A variational approach, preprint.

2. L. Boccardo, P. Drabek, D. Giachetti, and M. Kucera, Generalization of Fredholm alternative for nonlinear differential operators, Nonlinear Anal. 10 (1986), 1083-1103. 
3. M. del Pino, M. Elgueta, and R. Manásevich, A homotopic deformation along $p$ of a LeraySchauder degree result and existence for $\left(\left|u^{\prime}\right|^{p-2} u^{\prime}\right)^{\prime}+f(t, u)=0, u(0)=u(T)=0, \mathrm{~J}$. Differential Equations (1989).

4. Sturm's comparison theorem and a Hartman's type oscillation criterion for $\left(\left|u^{\prime}\right|^{p-2} u^{\prime}\right)^{\prime}+a(t)|u|^{p-2} u=0$, preprint.

5. P. Drabek, Ranges of a-homogeneous operators and their perturbations, Časopis Pěst. Mat. 105 (1980), 167-183.

6. M. Guedda and L. Veron, Bifurcation phenomena associated to the p-Laplace operator, Trans. Amer. Math. Soc. 310 (1988), 419-431.

School of Mathematics, University of Minnesota, Minneapolis, Minnesota 55455

Departamento de Matemáticas, F.C.F.M. Universidad de Chile, Casilla 170, Correo 3, Santiago, Chile 